\title{
EVALUACIÓN DE FACTORES QUE PUEDEN INFLUIR EN EL PROCESO DE SACARIFICACIÓN-FERMENTACIÓN SIMULTÁNEAS PARA LA PRODUCCIÓN DE ETANOL A PARTIR DE MATERIALES AMILÁCEOS
}

\author{
Bárbara Miranda Morales \\ Manuel E. Molina Córdoba
}

\begin{abstract}
Resumen
El objetivo general de esta investigación fue evaluar la posibilidad de realizar las etapas de sacarificación de almidón y fermentación de azúcares de manera simultánea, con el fin de reducir el tiempo del proceso global de producción de etanol a partir de materiales amiláceos. Para ello se estudiaron factores tales como: tipo y concentración de almidón, concentración de etanol, tiempo y temperatura de sacarificación, presencia de etanol y nutrientes $\left(\mathrm{K}_{2} \mathrm{HPO}_{4}, \mathrm{MgSO}_{4}\right.$ • $7 \mathrm{H}_{2} \mathrm{O}, \mathrm{NH}_{4} \mathrm{NO}_{3}$ y peptona) durante la etapa de hidrólisis del almidón, temperatura de fermentación.

Se encontró que el tipo de almidón y su concentración, no afecta significativamente el rendimiento de azúcares reductores. Además, que la presencia de etanol en concentraciones de $0 \% \mathrm{v} / \mathrm{v}$ hasta $12 \% \mathrm{v} / \mathrm{v}$ no afecta la actividad de la enzima AMG durante la sacarificación a temperaturas de $60{ }^{\circ} \mathrm{C}$ y $32{ }^{\circ} \mathrm{C}$. El tiempo de sacarificación empleado, sí afecta significativamente el rendimiento de azúcares reductores. La presencia de nutrientes en concentraciones usuales para una fermentación, no afecta la actividad de la enzima AMG durante la hidrólisis del almidón. Según los resultados de esta investigación, se concluyó que para aumentar el rendimiento de azúcares reductores, la mejor combinación de niveles es una temperatura de sacarificación de $60^{\circ} \mathrm{C}$ y un tiempo sacarificación de 2 h. Además, que las etapas de sacarificación y fermentación pueden realizarse de forma simultánea aún cuando se opere a $32{ }^{\circ} \mathrm{C}$. Los resultados de concentración de etanol obtenidos son comparables con valores de concentración de $(6,0$ a 7,5$) \%$ v/v usuales en la industria.
\end{abstract}

Palabras clave: Sacarificación, inhibición, enzimas, fermentación.

\begin{abstract}
The purpose of this research was to evaluate the possibility of performing the steps of saccharification and fermentation simultaneously, in order to reduce the time of production of ethanol from starch. Factors such as type and concentration of starch, concentration of ethanol, time and temperature of saccharification, presence of ethanol and nutrients $\left(\mathrm{K}_{2} \mathrm{HPO}_{4}, \mathrm{MgSO}_{4} \bullet 7 \mathrm{H}_{2} \mathrm{O}, \mathrm{NH}_{4} \mathrm{NO}_{3}\right.$ y peptone) were evaluated.

We found that the type of starch and its concentration does not affect significantly the yield of reducing sugars. Furthermore, the presence of ethanol at concentrations of $0 \% \mathrm{v} / \mathrm{v}$ to $12 \% \mathrm{v} / \mathrm{v}$ does not affect the activity of the AMG during the saccharification at $60{ }^{\circ} \mathrm{C}$ and $32{ }^{\circ} \mathrm{C}$. The time of saccharification affect significantly the production of reducing sugars. The presence of nutrients does not affect the activity of the enzyme AMG during starch hydrolysis. From these results, it was concluded that in order to increase the yield of reducing sugars, the best combination of temperature and time of saccharification was: $60{ }^{\circ} \mathrm{C}$ and $2 \mathrm{~h}$. Also, it was concluded that the saccharification and fermentation steps may take place simultaneously even when operating at $32{ }^{\circ} \mathrm{C}$. The results of concentration of ethanol obtained (6.0 to 7.5$) \% \mathrm{v} / \mathrm{v}$ are comparable to those values in industry.
\end{abstract}

Keywords: Saccharification, inhibition, enzymes, fermentation.

\section{INTRODUCCIÓN}

El transporte automotor constituye una de las actividades que mayor grado de contaminación ambiental genera, debido a los gases de escape emanados de los vehículos como producto de la combustión interna. El consumo de hidrocarburos en el 2005 en Costa Rica alcanzó los 16,6 
millones de barriles y se considera que en el año 2019, de seguir la tendencia actual, llegaría a 20 millones. La contaminación ambiental y la insostenible e inestable condición del mercado petrolero mundial, ha obligado a los Gobiernos a implementar durante los últimos años un plan de contingencia, dirigido especialmente a reducir el consumo de gasolina y desarrollar iniciativas para impulsar la producción nacional de biocombustibles (Harrison, 2007).

Con la finalidad de sustituir parcialmente la gasolina se ha orientado la investigación al uso de fuentes alternas y renovables para la producción de etanol (Assis, Paiva, Cabello y Días, 2010). Algunas de las fuentes que se han utilizado son tubérculos, raíces y granos (Masiero, Peretti, Trierweiler \& Trierweiler, 2014). Se estima que el rechazo de raíces y tubérculos en Costa Rica constituye aproximadamente un $20 \%$, convirtiéndose esto en una buena opción de aprovechamiento.

El almidón es un componente importante de productos tales como la papa, el maíz, el sagú, sorgo y yuca (Curvelo-Santana, Ehrhardt \& Tambourgi 2010). Duvernay, Chinn y Yencho (2013) investigaron la producción de etanol a partir de camote. También, Srichuwong et al. (2009) investigaron la obtención de etanol a partir de papa.

La hidrólisis del almidón es el paso inicial para obtener los azúcares que se utilizan en la fermentación. Este proceso puede realizarse por vía ácida o enzimática, ésta última consiste de dos etapas: licuefacción y sacarificación (Monsalve, Medina y Ruiz, 2006). El proceso de hidrólisis enzimática consiste en romper las moléculas de almidón hasta obtener glucosa utilizando dos enzimas, la $\alpha$-amilasa y luego una amiloglucosidasa (AMG). La glucosa obtenida es el sustrato en la etapa posterior de fermentación (Castaño, Cardona, Mejía y Acosta, 2011).

La licuefacción consiste en la hidrólisis parcial del almidón a dextrinas y es usualmente llevado a cabo en un tiempo de $2 \mathrm{~h}$, se utiliza la enzima $\alpha$-amilasa que se caracteriza por su termo-estabilidad (Wiseman, 1985), mientras que la sacarificación tarda de 12 h a 48 h dependiendo del grado de hidrólisis deseado. Para la sacarificación se usan las AMG, estas enzimas inician la hidrólisis de las dextrinas en los extremos no reductores de la molécula por las uniones $\alpha-1,4$, así como las uniones $\alpha-1,6$ originan- do dextrinas, maltosa y progresivamente avanza hasta liberar glucosa; la temperatura óptima debe oscilar entre los $60^{\circ} \mathrm{C}$ y $75^{\circ} \mathrm{C}$ (Meléndez, 2002).

Una vez obtenidos los azúcares a partir del almidón, estos se convierten a etanol por medio de una fermentación anaerobia, utilizando la Saccharomyces cereviseae. Para que el proceso de fermentación alcohólica sea lo más eficiente posible, es necesario controlar una serie de aspectos tales como: efecto del oxígeno, nutrientes, temperatura, $\mathrm{pH}$, concentración de la glucosa y efecto del etanol hacia las enzimas (Meléndez, 2002).

Investigadores como Vong Tsoi (1996), Meléndez (2002), Cunningham y López (1994) y Cubero (2005), orientaron sus trabajos a establecer condiciones de operación al utilizar diferentes fuentes de carbohidratos para la producción de etanol. González y Molina (2006) estudiaron los factores que afectan la hidrólisis enzimática y el proceso fermentativo del almidón de la papa (Solanum tuberosum) para la producción de etanol, obteniendo un rendimiento global de $0,0746 \mathrm{~kg}_{\text {etanol }} / \mathrm{kg}_{\text {sustrato }}$; en el proceso de hidrólisis utilizaron una $\alpha$-amilasa y posteriormente una AMG. Kiliç (2004) trabajando con una $\alpha$-amilasa encontró que la presencia de etanol en concentraciones desde $0 \% \mathrm{v} / \mathrm{v}$ a $20 \% \mathrm{v} / \mathrm{v}$ no afectan significativamente la acción de la enzima en la hidrólisis de almidón de arroz. López, Molina \& Huguet (2004) estudiaron la producción de etanol utilizando diferentes sustratos preparados a partir de banano, logrando un rendimiento de $0,0742 \mathrm{~L}_{\text {etanol }} / \mathrm{kg}_{\text {fruta }}$ completa.

El realizar el proceso de sacarificaciónfermentación de forma simultánea tiene ventajas tales como menor gasto en equipo, menor riesgo de contaminación, altas tasas de producción, y menor tiempo de producción del etanol. Además, presenta desventajas tales como que la hidrólisis y la fermentación deben llevarse a cabo bajo las mismas condiciones, cada proceso es llevado a cabo en un ambiente subóptimo (Sasaki, Kushiki, Asada \& Nakamura, 2014). Zhang et al. (2010) investigaron la sacarificación y fermentación simultánea de batata, considerando el proceso interesante desde el punto de vista económico y ambiental.

Esta investigación tiene por objetivo evaluar, a nivel de laboratorio, la posibilidad de realizar las etapas de sacarificación del 
almidón y fermentación de azúcares de manera simultánea, con el fin de reducir el tiempo del proceso global de producción de etanol a partir de materiales amiláceos.

\section{EQUIPO, MATERIALES Y METODO- LOGÍA EXPERIMENTAL}

\subsection{Materias primas}

Se utilizaron cuatro materiales amiláceos: almidón de maíz (SIGMA), almidón de yuca, fécula de maíz y sagú (Maranta arundinacea); estos últimos fueron adquiridos en un mercado local. A cada uno de los almidones se le determinó el contenido inicial de azúcares reductores.

\subsection{Enzimas}

Para realizar la hidrólisis del almidón se utilizaron enzimas de la casa fabricante Novo.

- $\quad \alpha$-amilasa (Termamyl 120 L): Se empleó para realizar la licuefacción; se recomienda un $\mathrm{pH}$ óptimo de 6,0-6,5 y la temperatura óptima de (93-107) ${ }^{\circ} \mathrm{C}$.

- Glucoamilasa (AMG 300L): Se usó en la etapa de sacarificación, el $\mathrm{pH}$ para esta enzima es de 4,5 y una de temperatura de $60{ }^{\circ} \mathrm{C}$.

\subsection{Métodos de análisis}

- Azúcares reductores: se utilizó el método de Nelson Somoghi (Southgate, 1976), la lectura de la absorbencia se realiza a una longitud de onda de $500 \mathrm{~nm}$ utilizando un espectrofotómetro Spectronic 20.

- Concentración de etanol: se utilizó el método de índice de refracción. Se realiza una curva de calibración para disoluciones de etanol en agua hasta un máximo de 10 $\% \mathrm{v} / \mathrm{v}$. El procedimiento consiste en filtrar el mosto fermentado, luego se toma una alícuota de $50 \mathrm{~mL}$ y traslada a un balón de $100 \mathrm{~mL}$, el cual se afora con agua destilada. La destilación se realiza hasta obtener un volumen de $50 \mathrm{~mL}$ de destilado y se determina el índice de refracción a una temperatura de $20{ }^{\circ} \mathrm{C}$ utilizando un refractómetro Abbé.

\subsection{Microorganismo}

Para la fermentación alcohólica se utilizó una cepa de Saccharomyces cereviseae proporcionada por el Laboratorio de Micología Médica de la Facultad de Microbiología de la Universidad de Costa Rica.

\subsection{Hidrólisis enzimática de los cuatro tipos de almidón}

Los objetivos de esta primer etapa fueron determinar si el tipo y concentración de almidón tienen un efecto significativo en el rendimiento de azúcares reductores durante la etapa de licuefacción y, determinar si la presencia de etanol durante la etapa de sacarificación del almidón afecta la acción de la enzima AMG para la producción de azúcares reductores, independientemente de la concentración y tipo de almidón.

\section{Licuefacción}

Para esta etapa se preparó, a nivel de erlenmeyer $(250 \mathrm{~mL})$, suspensiones de cada tipo de almidón a concentraciones de $(10,15,20$ y 25) $\% \mathrm{~m} / \mathrm{m}$, se reguló el $\mathrm{pH}$ a 6,5 , se adicionó $\mathrm{CaCl}_{2}$ a una concentración de 40 ppm y la enzima $\alpha$-amilasa a una concentración de $0,6 \mathrm{~mL} / \mathrm{kg}_{\text {Almidón }}$. Se llevó a cabo la licuefacción a $90^{\circ} \mathrm{C}$ utilizando un baño de temperatura controlada por un tiempo de 45 min. Para evaluar los resultados de esta etapa, los ensayos se distribuyeron en un experimento de dos factores sin repetición, donde los bloques fueron los tipos de almidón y los tratamientos fueron las concentraciones de almidón. Una vez transcurridos los $45 \mathrm{~min}$, se enfriaron las muestras y se bajó el $\mathrm{pH}$ a 4,5 utilizando $\mathrm{HCl}$ para desactivar la enzima $\alpha$-amilasa; se tomaron muestras para analizar azúcares reductores y se calcularon los rendimientos expresados como $\mathrm{mg}_{\mathrm{AR}} / \mathrm{mg}_{\text {Almidón }}$. 


\section{Sacarificación}

Los erlenmeyers se colocaron en una incubadora ajustada a una temperatura de $60{ }^{\circ} \mathrm{C}$ y una velocidad de agitación de $150 \mathrm{rev} / \mathrm{min}$. Una vez se alcanzó la temperatura, se adicionó la enzima amiloglucosidasa (AMG) a una concentración de $0,8 \mathrm{~mL} / \mathrm{kg}_{\text {Almidón }}$ y se adicionó etanol a diferentes concentraciones $(0,2,5$ y 8$)$ $\%$ v/v. Cada erlenmeyer fue cubierto con papel de aluminio para prevenir la pérdida de etanol durante el proceso. El tiempo de reacción se estableció en $6 \mathrm{~h}$, se tomaron muestras cada $2 \mathrm{~h}$, se centrifugaron para obtener un líquido claro sin sólidos y se analizaron para determinar azúcares reductores por el método de Nelson Somogyi; luego se calcularon los rendimientos. Para esta etapa de sacarificación los ensayos se distribuyeron en un diseño estadístico cuadrado latino de 4 x 4; las letras latinas corresponden a las concentraciones de etanol adicionado $(\mathrm{A}=$ $0 \% \mathrm{v} / \mathrm{v}$ etanol, $\mathrm{B}=2 \% \mathrm{v} / \mathrm{v}$ etanol, $\mathrm{C}=5 \% \mathrm{v} / \mathrm{v}$ etanol y $\mathrm{D}=8 \% \mathrm{v} / \mathrm{v}$ etanol). El diseño se muestra en el Tabla 1.

\subsection{Sacarificación del almidón de sagú a una temperatura de $32{ }^{\circ} \mathrm{C}$}

El objetivo de esta etapa fue determinar la capacidad de producción de azúcares fermentables por parte de la enzima AMG a una temperatura que no corresponde a la óptima $\left(60^{\circ} \mathrm{C}\right)$, y a su vez en presencia de diferentes concentraciones de etanol. El procedimiento seguido en esta etapa fue el mismo descrito en la sección 2.5, con las siguientes salvedades: sólo se empleó almidón de sagú y las suspensiones se prepararon a una concentración de $15 \% \mathrm{~m} / \mathrm{m}$, además la sacarificación se llevó a cabo a una temperatura de $32{ }^{\circ} \mathrm{C}$ por $30 \mathrm{~h}$. Se tomaron muestras a las (4, 18 y 30 ) h para su posterior análisis químico.

Se utilizó como variables experimentales: concentración de etanol: $(0,2,4,6,8,10$ y 12) $\%$ v/v, y tiempo de sacarificación: (4, 18 y 30) h. Como variable de respuesta se utilizan las mismas de las etapas previas. Para evaluar el objetivo de esta etapa experimental, se propuso un experimento de dos factores sin repetición, donde los bloques fueron los tiempos de sacarificación y los tratamientos fueron las concentraciones de etanol.

\subsection{Presencia de nutrientes durante la hidrólisis del almidón de sagú}

El objetivo de esta etapa fue determinar si la presencia de nutrientes, usualmente utilizados durante la fermentación alcohólica, tiene algún efecto sobre la actividad de las enzimas $\alpha$-amilasa y AMG durante la etapa de hidrólisis.

Se establecieron como variables fijas: temperatura de sacarificación $\left(60{ }^{\circ} \mathrm{C}\right)$, concentración de las suspensiones de almidón de sagú $(15 \% \mathrm{~m} / \mathrm{m})$, microorganismo usado para fermentación (Saccharomyces cerevisiae), temperatura de fermentación $\left(32{ }^{\circ} \mathrm{C}\right)$; tiempo de sacarificación (24h) y tiempo de fermentación (42 h). La variable experimental considerada fue la presencia de los nutrientes. Las variables de respuesta fueron: a) concentración de azúcares reductores y rendimientos b) concentración de etanol.

Se prepararon seis suspensiones de almidón, a los cuales se les agregó $\mathrm{CaCl} 2$ (40 ppm). A tres de las muestras se les adicionaron los nutrientes (ver Tabla 2), se homogenizaron y se reguló el $\mathrm{pH}$ a 6,5; seguidamente se agregó la enzima $\alpha$-amilasa $\left(0,6 \mathrm{~mL} / \mathrm{kg}_{\text {Almidón }}\right)$. Las muestras se mantuvieron en un baño a $90{ }^{\circ} \mathrm{C}$ durante $45 \mathrm{~min}$, luego las muestras fueron removidas del baño y enfriadas a temperatura ambiente, se tomó una parte de cada muestra para su posterior análisis. Luego se procedió a regular el $\mathrm{pH}$ a 4,5 y se adicionó la enzima $\mathrm{AMG}\left(0,8 \mathrm{~mL} / \mathrm{kg}_{\text {Almidón }}\right)$. Las muestras se introdujeron en una incubadora a $60{ }^{\circ} \mathrm{C}$ por 24 h. Finalizado el tiempo de sacarificación, las muestras se enfriaron hasta temperatura ambiente y se analizó azúcares reductores.

Concluida la sacarificación, se agregaron los nutrientes (ver Tabla 2) a las tres suspensiones a las que no se les había agregado durante la etapa de hidrólisis. Se añadió la levadura en un $10 \%$ del volumen del sustrato y se llevó a cabo la fermentación durante $42 \mathrm{~h}$. Transcurrido este tiempo se tomaron muestras de $60 \mathrm{~mL}$ y se centrifugaron; luego se determinó la concentración de etanol y concentración de azúcares reductores. 
Los resultados se analizaron mediante una prueba t de Student, para la comparación de dos tratamientos que corresponden a la presencia y ausencia de nutrientes. Para cada uno de los tratamientos se realizaron 2 repeticiones.

\subsection{Hidrólisis de almidón a temperaturas de $32{ }^{\circ} \mathrm{C}$ y $60{ }^{\circ} \mathrm{C}$}

El objetivo de esta etapa fue comparar la producción de azúcares reductores a partir de sustratos

Tabla 1. Diseño de cuadrado latino para la etapa de sacarificación del almidón

\begin{tabular}{ccccc}
\hline $\begin{array}{c}\text { Concentración de } \\
\text { almidón } \\
(\% \mathbf{m} / \mathbf{m})\end{array}$ & Yuca & Sagú & Maíz de almidón & Fécula de maíz \\
\cline { 2 - 5 } & A & B & C & D \\
15 & B & A & D & C \\
20 & C & D & A & B \\
25 & D & C & B & A \\
\hline
\end{tabular}

Tabla 2. Nutrientes adicionados en la etapa de hidrólisis

\begin{tabular}{cc}
\hline Nutriente & Concentración $\mathbf{( g / \mathbf { L } )}$ \\
\hline $\mathrm{K}_{2} \mathrm{HPO}_{4}$ & 1,0 \\
$\mathrm{MgSO}_{4}$ & 0,25 \\
$\mathrm{NH}_{4} \mathrm{NO}_{3}$ & 1,5 \\
Peptona & 1,0 \\
\hline
\end{tabular}

Tabla 3. Resultados de rendimiento de azúcares reductores $\left(\mathrm{mg}_{\mathrm{AR}} / \mathrm{mg}_{\mathrm{Almidón}}\right)$ al final de la etapa de licuefacción

\begin{tabular}{ccccc}
\hline $\begin{array}{c}\text { Concentración de } \\
\text { almidón } \\
(\mathbf{\%} \mathbf{~ m} / \mathbf{m})\end{array}$ & Yuca & Sagú & Maíz & Fécula de maíz \\
\cline { 2 - 4 } & 0,86 & 1,17 & 1,58 & 1,45 \\
10 & 0,67 & 1,65 & 1,52 & 0,47 \\
15 & 0,80 & 0,80 & 1,11 & 0,87 \\
20 & 1,08 & 1,07 & 0,61 & 1,24 \\
\hline
\end{tabular}


preparados bajo dos temperaturas y tres tiempos de sacarificación distintos, y evaluar la producción de etanol a partir de cada uno de los sustratos.

La etapa de licuefacción se llevó a cabo de la manera descrita en la sección 2.5, utilizando una concentración de almidón de sagú de $15 \%$ $\mathrm{m} / \mathrm{m}$. Para la etapa de sacarificación, una vez agregada la enzima $\mathrm{AMG}$, seis de los erlenmeyers se introdujeron en una incubadora a $60{ }^{\circ} \mathrm{C}$ y seis se colocaron en un baño a $32{ }^{\circ} \mathrm{C}$. Al cabo de 1 $\mathrm{h}$ de sacarificación, se sacó un erlenmeyer y su duplicado, de cada uno de los baños y se enfriaron para detener la acción de la enzima AMG. Se procedió de igual forma a las $2 \mathrm{~h} \mathrm{y} 4 \mathrm{~h}$; se tomaron muestras para analizar azúcares reductores.

Finalizada la etapa de sacarificación, se agregaron los nutrientes (ver Tabla 2) y la levadura, ésta última se agregó en un $10 \%$ del volumen del sustrato. La fermentación se llevó a cabo durante $42 \mathrm{~h}$ a una temperatura de $32{ }^{\circ} \mathrm{C}$. Transcurrido este tiempo se tomaron muestras de $60 \mathrm{~mL}$ y se centrifugaron, luego se determinó la concentración de etanol y la concentración de azúcares reductores. Los resultados se analizaron mediante un análisis de varianza de dos factores con repetición.

\subsection{Hidrólisis enzimática y fermentación del almidón de sagú en un Reactor de 5 L}

El objetivo de esta etapa fue medir la variación en la producción de etanol y azúcares reductores respecto al tiempo. Para esta parte se utilizó una concentración de almidón de sagú de $20 \% \mathrm{~m} / \mathrm{m}$. Para tal propósito se colocó una masa de $800 \mathrm{~g}$ de almidón de sagú en un beaker de $4 \mathrm{~L}$ y se llevó con agua destilada a una masa total de $4 \mathrm{~kg}$. Con dicha suspensión se realizó la licuefacción y la sacarificación según el procedimiento descrito en la sección 2.5. La sacarificación se llevó a cabo durante 3 h, al final de este tiempo se tomaron muestras para su posterior análisis.

Para realizar la fermentación a mayor escala, se utilizó un reactor Electrolab de un volumen efectivo de $5 \mathrm{~L}$, que cuenta con puertos para toma de muestras, introducción de inóculo, sustrato, además cuenta con sistemas de control de temperatura, $\mathrm{pH}$ y medición de oxígeno disuelto. Dispone de una interfase a una computadora para programar las condiciones de operación y registro. Del sustrato hidrolizado se tomaron $150 \mathrm{~mL}$ para la preparación del inóculo de $S$. cereviseae y el resto se pasó al fermentador y se le adicionaron los nutrientes (ver Tabla 2). Se adicionó el inóculo a una concentración aproximada de $10 \%$ en volumen, este momento se registró como el tiempo cero y se dejó la fermentación por un tiempo de $28 \mathrm{~h}$, con una agitación de $75 \mathrm{rev} / \mathrm{min}$ y una temperatura de $32{ }^{\circ} \mathrm{C}$. A intervalos de tiempo de $2 \mathrm{~h}$ a $3 \mathrm{~h}$ se tomaron muestras para determinar azúcares reductores y concentración de etanol. Este procedimiento se realizó por duplicado.

\section{RESULTADOS Y DISCUSIÓN}

\subsection{Hidrólisis enzimática de los cuatro tipos de almidón.}

La hidrólisis enzimática se realizó en dos etapas:

\section{Licuefacción}

Los resultados de rendimiento obtenidos en esta etapa se presentan en la Tabla 3 y el análisis estadístico se presenta en la Tabla 4. Se observa que la magnitud del valor $\mathrm{F}_{\text {exp }}$ para las distintas fuentes de variación es inferior al valor teórico de $\mathrm{F}_{(3 / 9)}=3,86$ para un $95 \%$ de confianza en una prueba de dos colas; se encuentra que ni la concentración ni el tipo de almidón proporcionan resultados significativamente diferentes en sus distintos niveles.

\section{Sacarificación}

Para esta etapa de sacarificación se tomaron muestras a las $(2,4$ y 6$) \mathrm{h}$. A las $2 \mathrm{~h}$ el material de cada ensayo es muy viscoso puesto que la enzima $\alpha$-amilasa no ha desdoblado suficientemente el almidón y por consiguiente es difícil la toma de muestras homogéneas; para efecto de análisis estadístico solo se consideraron los resultados para los otros tiempos. Los datos de rendimientos para las muestras tomadas a las $4 \mathrm{~h} \mathrm{y} 6 \mathrm{~h}$ de sacarificación se muestran en las Tablas 5 y 6 . 
Tabla 4. Prueba estadística F aplicada a los datos de rendimiento para la etapa de licuefacción

\begin{tabular}{cccccc}
\hline Fuente de variación & $\begin{array}{c}\text { Suma de Cua- } \\
\text { drados }\end{array}$ & $\begin{array}{c}\text { Grados de } \\
\text { Libertad }\end{array}$ & $\begin{array}{c}\text { Promedio de los } \\
\text { cuadros }\end{array}$ & $\mathbf{F}_{\text {exp }}$ & F $_{\text {teo }}$ \\
\hline $\begin{array}{c}\text { Concentración de } \\
\text { Almidón }\end{array}$ & 0,292 & 3 & 0,097 & 0,66 & 3,86 \\
Tipo de almidón & 0,316 & 3 & 0,105 & 0,71 & 3,86 \\
Error & 1,335 & 9 & 0,148 & \\
Total & 1,943 & 15 & & \\
\hline
\end{tabular}

Tabla 5. Rendimientos a las 4 h de hidrólisis enzimática de los cuatro tipos de almidón empleados

\begin{tabular}{ccccc}
\hline $\begin{array}{c}\text { Concentración } \\
\text { de almidón }\end{array}$ & \multicolumn{4}{c}{ Tipo de almidón } \\
\cline { 2 - 5 }$(\mathbf{\%} \mathbf{~ m} \mathbf{m})$ & Yuca & Sagú & Maíz & $\begin{array}{c}\text { Fécula de } \\
\text { Maíz }\end{array}$ \\
\hline \multirow{2}{*}{10} & $\mathrm{~A}$ & $\mathrm{~B}$ & $\mathrm{C}$ & $\mathrm{D}$ \\
& 4,36 & 5,23 & 11,92 & 3,90 \\
15 & $\mathrm{~B}$ & $\mathrm{~A}$ & $\mathrm{D}$ & $\mathrm{C}$ \\
& 4,61 & 4,26 & 3,39 & 5,55 \\
20 & $\mathrm{C}$ & $\mathrm{D}$ & $\mathrm{A}$ & $\mathrm{B}$ \\
& 2,61 & 1,67 & 6,40 & 4,02 \\
25 & $\mathrm{D}$ & $\mathrm{C}$ & $\mathrm{B}$ & $\mathrm{A}$ \\
& 2,48 & 3,75 & 4,33 & 2,00 \\
\hline
\end{tabular}

Tabla 6. Rendimientos a las 6 h de hidrólisis enzimática de los cuatro tipos de almidón empleados

\begin{tabular}{|c|c|c|c|c|}
\hline \multirow{2}{*}{$\begin{array}{c}\text { Concentración } \\
\text { de almidón } \\
(\% \mathbf{m} / \mathbf{m})\end{array}$} & \multicolumn{4}{|c|}{ Tipo de almidón } \\
\hline & Yuca & Sagú & Maíz & $\begin{array}{c}\text { Fécula de } \\
\text { Maíz }\end{array}$ \\
\hline \multirow{2}{*}{10} & A & B & $\mathrm{C}$ & $\mathrm{D}$ \\
\hline & 5,56 & 11,19 & 12,84 & 3,71 \\
\hline \multirow{2}{*}{15} & B & A & $\mathrm{D}$ & $\mathrm{C}$ \\
\hline & 3,74 & 4,21 & 3,68 & 5,22 \\
\hline \multirow{2}{*}{20} & $\mathrm{C}$ & $\mathrm{D}$ & A & B \\
\hline & 3,91 & 2,32 & 7,50 & 3,15 \\
\hline \multirow{2}{*}{25} & $\mathrm{D}$ & $\mathrm{C}$ & B & A \\
\hline & 6,07 & 5,56 & 4,63 & 5,65 \\
\hline
\end{tabular}


Tabla 7. Resultados de la prueba estadística $\mathrm{F}$ aplicada a los datos de rendimiento obtenidos a las 4 h y 6 h de sacarificación

\begin{tabular}{ccccc}
\hline Fuente de variación & $\begin{array}{c}\text { Grados de } \\
\text { Libertad }\end{array}$ & $\begin{array}{c}\mathbf{F}_{\text {exp }} \\
\mathbf{( 4 ~ h )}\end{array}$ & $\begin{array}{c}\mathbf{F}_{\text {exp }} \\
(\mathbf{6 h})\end{array}$ & $\mathbf{F}_{\text {teo }}$ \\
\hline $\begin{array}{c}\text { Concentración de } \\
\text { Almidón }\end{array}$ & 3 & 2,68 & 2,23 & 4,76 \\
Tipo de almidón & 3 & 2,70 & 0,88 & 4,76 \\
Concentración de etanol & 3 & 2,19 & 0,87 & 4,76 \\
Error & 6 & & & \\
\hline
\end{tabular}

En la Tabla 7 se presentan los resultados del análisis estadístico para los datos de rendimiento obtenidos a las 4 h y 6 h de sacarificación. La magnitud del valor de Fexp mostrado en la Tabla 7 para las distintas fuentes de variación tanto a las $4 \mathrm{~h}$ como a las $6 \mathrm{~h}$, resultó menor al valor teórico de $\mathrm{F}(3 / 6)=4,76$ para un $95 \%$ de confianza en una prueba de dos colas, por lo que se encuentra que ninguna fuente de variación proporciona resultados significativamente diferentes en sus distintos niveles.

Estos resultados permiten establecer que se puede trabajar indistintamente con cualquiera de los cuatro tipos de almidón. Cualitativamente se observó que el almidón de yuca es el que tiende a formar mayor cantidad de grumos y el jarabe obtenido adquiere un color café claro como consecuencia de reacciones de pardeamiento no enzimático, por el contrario, las suspensiones de almidón de maíz, de fécula de maíz y de sagú resultan más sencillas de trabajar y el color de las suspensiones es de blanco a un blanco hueso. Atendiendo a lo anterior se escogió trabajar con almidón de sagú para las siguientes etapas experimentales.

En cuanto a la concentración de almidón, los resultados muestran que se puede trabajar con cualquiera de las concentraciones probadas sin diferencia significativa en los rendimientos obtenidos, asegurando que al adicionar mayor cantidad de almidón se obtendrá mayor cantidad de azúcares reductores. Se establece trabajar con una concentración de $15 \% \mathrm{~m} / \mathrm{m}$ en etapas posteriores. La concentración de etanol adicionada a cada ensayo sometido a sacarificación no presenta una diferencia significativa en la actividad de la enzima AMG, esto es consistente con resultados obtenidos por Kiliç (2004), quienes trabajaron con $\alpha$-amilasa y encontraron que la presencia de etanol en concentraciones de $0 \% \mathrm{v} / \mathrm{v}$ hasta $20 \%$ v/v no afectan significativamente la acción de la enzima en la hidrólisis de almidón de arroz.

\subsection{Sacarificación del almidón de sagú a una temperatura de $32{ }^{\circ} \mathrm{C}$}

Las variables estudiadas en este diseño experimental fueron la concentración de etanol y el tiempo de sacarificación en los niveles presentados en la Tabla 8, a la vez se muestran los resultados de rendimiento de esta hidrólisis.

Los resultados del análisis estadístico se muestran en la Tabla 9. Puesto que el valor de $\mathrm{F}_{\exp }$ observado para los bloques, es menor al $F_{\text {teo }}$ para un nivel de significancia del $5 \%$, se encuentra que el tiempo de sacarificación sí afecta los resultados de rendimiento de forma significativa. En contraste, para los tratamientos, los resultados muestran que la concentración de etanol no afecta de forma significativa la acción de la enzima AMG durante la etapa de hidrólisis del almidón. Estos resultados dejan establecido que la presencia de etanol en las concentraciones estudiadas no afecta la acción de la AMG durante la sacarificación.

\subsection{Presencia de nutrientes durante la hidrólisis del almidón de sagú}

Esta etapa se realizó por triplicado y los resultados de concentración de azúcares reductores y rendimiento se muestran en la Tabla 10.

El análisis estadístico de los rendimientos, se efectuó mediante una prueba estadística relativa a la diferencia entre dos medias utilizando el 
Tabla 8. Rendimientos $\left(\mathrm{mg}_{\mathrm{AR}} / \mathrm{mg}_{\text {Almidón }}\right)$ obtenidos durante la sacarificación del almidón de sagú a una temperatura de $32{ }^{\circ} \mathrm{C}$

\begin{tabular}{ccccc}
\hline \multirow{2}{*}{$\begin{array}{c}\text { Concentración de etanol } \\
(\mathbf{\%} \mathbf{v} / \mathbf{v})\end{array}$} & $\mathbf{0}$ & $\mathbf{4}$ & $\mathbf{1 8}$ & $\mathbf{3 0}$ \\
\cline { 2 - 5 } $\mathbf{0}$ & 1,66 & 4,38 & 7,30 & 12,25 \\
$\mathbf{2}$ & 1,52 & 4,71 & 6,98 & 12,78 \\
$\mathbf{4}$ & 1,63 & 3,58 & 6,80 & 7,74 \\
$\mathbf{6}$ & 1,58 & 3,56 & 7,01 & 7,30 \\
$\mathbf{8}$ & 1,49 & 3,03 & 6,32 & 9,72 \\
$\mathbf{1 0}$ & 1,56 & 3,29 & 3,88 & 8,17 \\
$\mathbf{1 2}$ & 1,70 & 3,28 & 5,03 & 7,02 \\
\hline
\end{tabular}

Tabla 9. Prueba estadística F aplicada a los datos de rendimiento obtenidos durante la hidrólisis del almidón de sagú

\begin{tabular}{cccccc}
\hline Fuente de variación & $\begin{array}{c}\text { Suma de } \\
\text { cuadrados }\end{array}$ & $\begin{array}{c}\text { Grados de } \\
\text { Libertad }\end{array}$ & $\begin{array}{c}\text { Promedio de los } \\
\text { Cuadrados }\end{array}$ & F $_{\text {exp }}$ & F $_{\text {teo }}$ \\
\hline $\begin{array}{c}\text { Concentración de } \\
\text { Almidón }\end{array}$ & 20,55 & 6 & 3,42 & 2,44 & 2,66 \\
Tiempo de Sacarificación & 230,63 & 3 & 76,86 & 54,64 & 3,16 \\
Error & 25,32 & 18 & 1,40 & & \\
Total & 276,51 & 27 & & \\
\hline
\end{tabular}

estadístico $t$ de Student. Las hipótesis para este análisis fueron:

$\mathrm{H}_{0}: \mu$ con nutrientes $=\mu$ sin nutrientes

$\mathrm{H}_{1}: \mu$ con nutrientes $\neq \mu$ sin nutrientes

Los resultados de la prueba $t$ de Student para los datos de rendimiento se encuentran en la Tabla 11. Los datos muestran que el $\mathrm{t}_{\mathrm{cal} \text { AR }} \mathrm{de}$ la etapa de licuefacción es $-0,379$, valor que se encuentra dentro del intervalo (-2, 776 a 2,776), lo que implica que no existe una diferencia estadística entre los promedios, es decir la actividad de la enzima $\alpha$-amilasa no se ve afectada por la presencia de nutrientes. De igual forma ocurre para la etapa de sacarificación, dado que $-2,776<1,195<2,776$, por lo que se acepta la hipótesis nula.

Según los resultados mostrados en la Tabla 10, la concentración de azúcares reductores obtenida al final de la sacarificación para cada una de las muestras, se encuentra dentro del ámbito de concentraciones moderadas ( 3 a 100) g/L que permiten la formación de etanol, según Meléndez (2002). Por lo que finalizada esta etapa, se adicionaron nutrientes a aquellas muestras que no los contenían y se procedió a realizar su fermentación inoculando $S$. cerevisiae. Concluida la fermentación se tomaron muestras y se analizó azúcares reductores y contenido de etanol, dichos resultados se muestran en la Tabla 12. Los resultados de concentración de etanol obtenidos están dentro de un ámbito aceptable, basados en que industrialmente, utilizando otras fuentes de carbono, logran tener en sus mostos una concentración de etanol de $(6,0$ a 7,5) \% v/v. La concentración de azúcares reductores residuales presentes en cada una de las muestras fue relativamente alta por lo que es posible aumentar más la conversión a etanol. 
Tabla 10. Valores de concentración de azúcares reductores y rendimiento obtenidos durante la etapa de hidrólisis del almidón de sagú en presencia de nutrientes

\begin{tabular}{|c|c|c|c|c|c|}
\hline \multirow[b]{2}{*}{ Tratamiento } & \multirow[b]{2}{*}{$\begin{array}{c}\text { No. de } \\
\text { muestra }\end{array}$} & \multicolumn{2}{|c|}{ Final de licuefacción } & \multicolumn{2}{|c|}{ Final de sacarificación } \\
\hline & & $\begin{array}{c}\text { Conc. AR } \\
(\mathrm{mg} / \mathrm{L})\end{array}$ & $\begin{array}{c}\text { Rendimiento } \\
\left(\mathrm{mg}_{\mathrm{AR}} / \mathrm{mg}_{\text {Almidón }}\right)\end{array}$ & $\begin{array}{c}\text { Conc. AR } \\
(\mathrm{mg} / \mathrm{L})\end{array}$ & $\begin{array}{c}\text { Rendimiento } \\
\left(\mathrm{mg}_{\mathrm{AR}} / \mathrm{mg}_{\text {Almidón }}\right)\end{array}$ \\
\hline \multirow{3}{*}{ Con nutrientes } & 1 & 2118,12 & 1,20 & 15136,96 & 8,58 \\
\hline & 2 & 2219,57 & 1,26 & 15151,45 & 8,59 \\
\hline & 3 & 2212,32 & 1,25 & 15622,46 & 8,85 \\
\hline \multirow{3}{*}{ Sin nutrientes } & 4 & 2139,86 & 1,21 & 14825,36 & 8,40 \\
\hline & 5 & 2226,81 & 1,26 & 15376,09 & 8,71 \\
\hline & 6 & 2234,06 & 1,27 & 14847,10 & 8,41 \\
\hline
\end{tabular}

Nota: AR: Azúcares Reductores

Tabla 11. Prueba estadística $t$ de Student para la etapa de licuefacción y sacarificación

\begin{tabular}{ccc}
\hline Parámetro & Final de licuefacción & Final de sacarificación \\
$\mathrm{t}_{\mathrm{cal} \text { AR }}$ & $-0,379$ & 1,195 \\
$\mathrm{t}$ tab $95 \%$ & 2,776 & 2,776 \\
Grados de libertad & 4 & 4 \\
\hline
\end{tabular}

Tabla 12. Concentración de azúcares reductores y etanol obtenidos al final de 42 h de fermentación durante la etapa de adición de nutrientes en la hidrólisis del almidón de sagú

\begin{tabular}{ccc}
\hline No. de muestra & $\begin{array}{c}\text { Concentración de azúcares } \\
\text { reductores residuales } \\
(\mathbf{m g} / \mathbf{L})\end{array}$ & $\begin{array}{c}\text { Concentración de etanol } \\
(\% \mathbf{\%} / \mathbf{v})\end{array}$ \\
\hline 1 & 7901,45 & 6,00 \\
2 & 18162,32 & 7,00 \\
3 & 13988,41 & 6,50 \\
5 & 12133,33 & 5,25 \\
6 & 11727,54 & 5,50 \\
\hline
\end{tabular}


Tabla 13. Rendimientos $\left(\mathrm{mg}_{\mathrm{AR}} / \mathrm{mg}_{\text {Almidón }}\right)$ obtenidos durante la etapa de hidrólisis del almidón de sagú a temperaturas de $60{ }^{\circ} \mathrm{C}$ y $32{ }^{\circ} \mathrm{C}$

\begin{tabular}{|c|c|c|c|c|c|}
\hline \multirow{2}{*}{$\begin{array}{l}\text { Temperatura de } \\
\text { sacarificación } \\
\left({ }^{\circ} \mathbf{C}\right)\end{array}$} & \multirow[t]{2}{*}{ No. de muestra } & \multicolumn{4}{|c|}{$\begin{array}{c}\text { Tiempo de sacarificación } \\
\text { (h) }\end{array}$} \\
\hline & & $\mathbf{0}$ & 1 & 2 & 4 \\
\hline \multirow{7}{*}{60} & 1 & 1,18 & 1,52 & - & - \\
\hline & 1.1 & 1,17 & 1,50 & - & - \\
\hline & 2 & 1,20 & - & 5,06 & - \\
\hline & & & & & \\
\hline & 2.1 & 1,16 & - & 5,07 & - \\
\hline & 3 & 1,06 & - & - & 5,40 \\
\hline & 3.1 & 1,17 & - & - & 5,26 \\
\hline \multirow{6}{*}{32} & 1 & 1,17 & 1,21 & - & - \\
\hline & 1.1 & 1,16 & 1,19 & - & - \\
\hline & 2 & 1,21 & - & 4,28 & - \\
\hline & 2.1 & 1,05 & - & 4,49 & - \\
\hline & 3 & 1,19 & - & - & 4,79 \\
\hline & 3.1 & 1,27 & - & - & 4,67 \\
\hline
\end{tabular}

\subsection{Hidrólisis de almidón a temperaturas de $32{ }^{\circ} \mathrm{C} \mathrm{y} 60{ }^{\circ} \mathrm{C}$}

El propósito de esta etapa fue evaluar la producción de azúcares reductores a partir de sustratos preparados bajo temperaturas y tiempos de sacarificación distintos, y evaluar la producción de etanol a partir de cada uno de los sustratos. Las pruebas se realizaron por duplicado y los resultados de rendimiento obtenidos se muestran en la Tabla 13.

Los resultados presentados en la Tabla 13 se analizaron por medio de un análisis de varianza de dos factores con repetición, este se presenta en la Tabla 14, en el cual al comparar el valor de $F_{\text {exp }}$ para las distintas fuentes de variación con el valor de $\mathrm{F}_{(5 / 8)}=5,32$ para la temperatura de sacarificación y $\mathrm{F}_{(3 / 8)}=4,07$ para el tiempo de sacarificación, se halla que ambas fuentes de variación proporcionan resultados significativamente diferentes en sus distintos niveles. Los resultados muestran que tanto la temperatura como el tiempo de sacarificación son factores muy importantes en el proceso de hidrólisis enzimática. Además, se encuentra que el efecto de interacción entre las variables de temperatura y tiempo de sacarificación es significativo (su análisis no se presenta).

En la Figura 1 se aprecia que al aumentar el tiempo de sacarificación, también aumenta el rendimiento de azúcares reductores, tanto a $32{ }^{\circ} \mathrm{C}$ como a $60{ }^{\circ} \mathrm{C}$, presentando un aumento mayor en el intervalo de $1 \mathrm{~h}$ a $2 \mathrm{~h}$ de sacarificación. Dado que el interés es aumentar el rendimiento de azúcares reductores, la mejor combinación de niveles es una temperatura de sacarificación de $60{ }^{\circ} \mathrm{C}$ y un tiempo de $2 \mathrm{~h}$.

Luego de finalizada la etapa de hidrólisis, la concentración de azúcares reductores se encuentra entre $(8000$ y 9500$)$ mg/L, estos valores están 
Tabla 14. Prueba estadística F aplicada a los datos de rendimiento obtenidos durante la etapa de hidrólisis enzimática del almidón de sagú

\begin{tabular}{cccccc}
\hline Fuente de variación & $\begin{array}{c}\text { Suma de } \\
\text { cuadrados }\end{array}$ & $\begin{array}{c}\text { Grados de } \\
\text { Libertad }\end{array}$ & $\begin{array}{c}\text { Promedio de los } \\
\text { Cuadrados }\end{array}$ & $\mathbf{F}_{\text {exp }}$ & F $_{\text {teo }}$ \\
\hline $\begin{array}{c}\text { Temperatura de sacari- } \\
\text { ficación }\end{array}$ & 0,618 & 1 & 0,618 & 118,19 & 5,32 \\
Tiempo de Sacarificación & 52,56 & 3 & 17,52 & 3349,23 & 4,07 \\
Interacción & 0,302 & 3 & 0,101 & 19,27 & 4,07 \\
Dentro del grupo & 0,042 & 8 & 0,0052 & & \\
Total & 53,52 & 15 & & & \\
\hline
\end{tabular}

dentro del ámbito de concentraciones moderadas que inhiben la respiración del microorganismo y aseguran la formación de etanol (Meléndez, 2002). Sin embargo, los valores de concentración de etanol obtenidos fueron bajos (valores no mostrados). Una posible explicación para esto podría ser que la levadura empleada no creció lo suficiente y por tanto la cantidad de levadura agregada a las muestras fue poca. La concentración de azúcares reductores residuales presentes en dichas muestras, es relativamente alta por lo que sería posible aumentar aún más la conversión a etanol.

\subsection{Hidrólisis enzimática y fermentación del almidón de sagú en un Reactor de $5 \mathrm{~L}$}

Con el fin de aplicar los resultados encontrados en las etapas anteriores, se realizó una hidrólisis y fermentación a mayor escala, utilizando el reactor Electrolab con una capacidad máxima de $5 \mathrm{~L}$. La etapa de sacarificación tuvo una duración de $3 \mathrm{~h}$ y la fermentación de aproximadamente $28 \mathrm{~h}$. El procedimiento se realizó por duplicado. Para esta etapa se determinó la variación en la concentración de etanol y de azúcares reductores con el tiempo, en la Figura 2 se muestran los resultados.

En la Figura 2a se muestra la variación en la concentración de etanol con el tiempo, hasta las 28 h de fermentación. Se observa para la corrida 1 que la concentración de etanol crece hasta las $23 \mathrm{~h}$, después de lo cual se mantiene a una concentración constante de $6,75 \% \mathrm{v} / \mathrm{v}$, el cual es un valor que alcanza el $70 \%$ de la capacidad para producir etanol que tienen este tipo de levaduras, además, dicho valor es similar a los obtenidos al realizar el proceso en erlenmeyer de $250 \mathrm{~mL}$.

En la Figura $2 b$ se observa la variación en la concentración de azúcares reductores con el tiempo. Para la corrida 1, al comenzar la fermentación existe una concentración de azúcares de $57062,80 \mathrm{mg} / \mathrm{L}$, lo cual es suficiente para que pueda iniciar dicho proceso. A las $3 \mathrm{~h}$ se muestra un aumento hasta 63000 $\mathrm{mg} / \mathrm{L}$, por lo que la enzima AMG ha continuado trabajando. En el período de las 23 h a las 28 $\mathrm{h}$, la concentración de azúcares se mantiene constante, lo que coincide con la zona plana de producción de etanol.

Para dar validez a los resultados obtenidos, el proceso se realizó por duplicado, y como se muestra en la Figura 2, la repetición sigue un comportamiento muy similar tanto para los azúcares reductores como para el etanol.

\section{CONCLUSIONES Y RECOMENDACIONES}

1. No se presenta una diferencia significativa en el rendimiento de azúcares reductores debido al tipo de almidón usado, por lo se puede trabajar indistintamente con cualquiera de los cuatro tipos de almidón probados.

2. Los diferentes niveles de concentración de etanol adicionados a los ensayos durante la etapa de sacarificación no ejercen un efecto significativo en la actividad de la enzima AMG para la producción de azúcares reductores. 


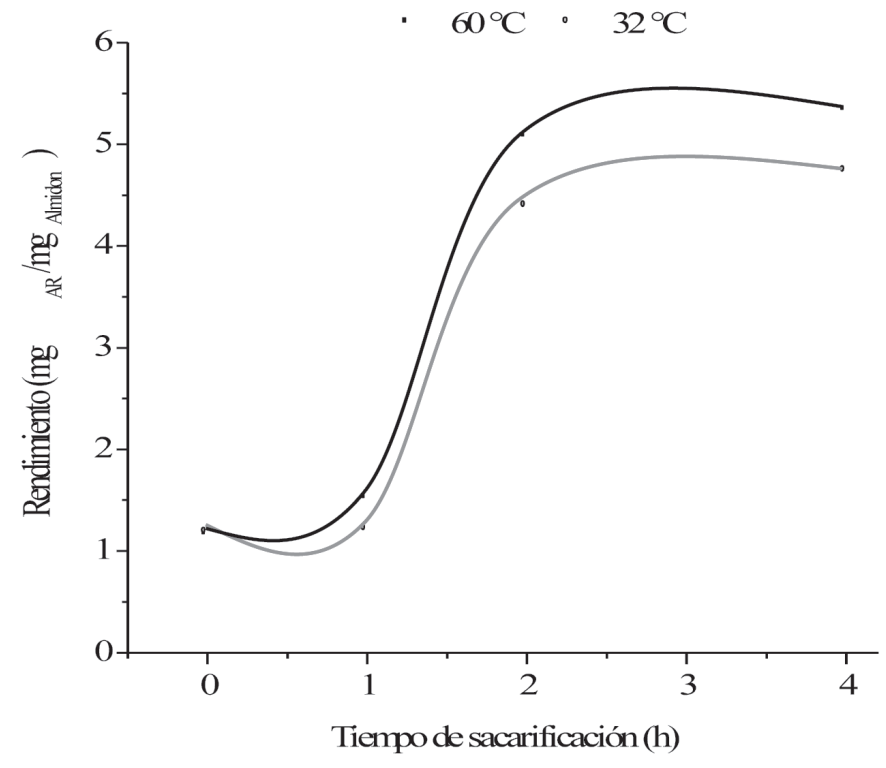

Figura 1. Representación del efecto de interacción entre los factores de tiempo y temperatura de sacarificación
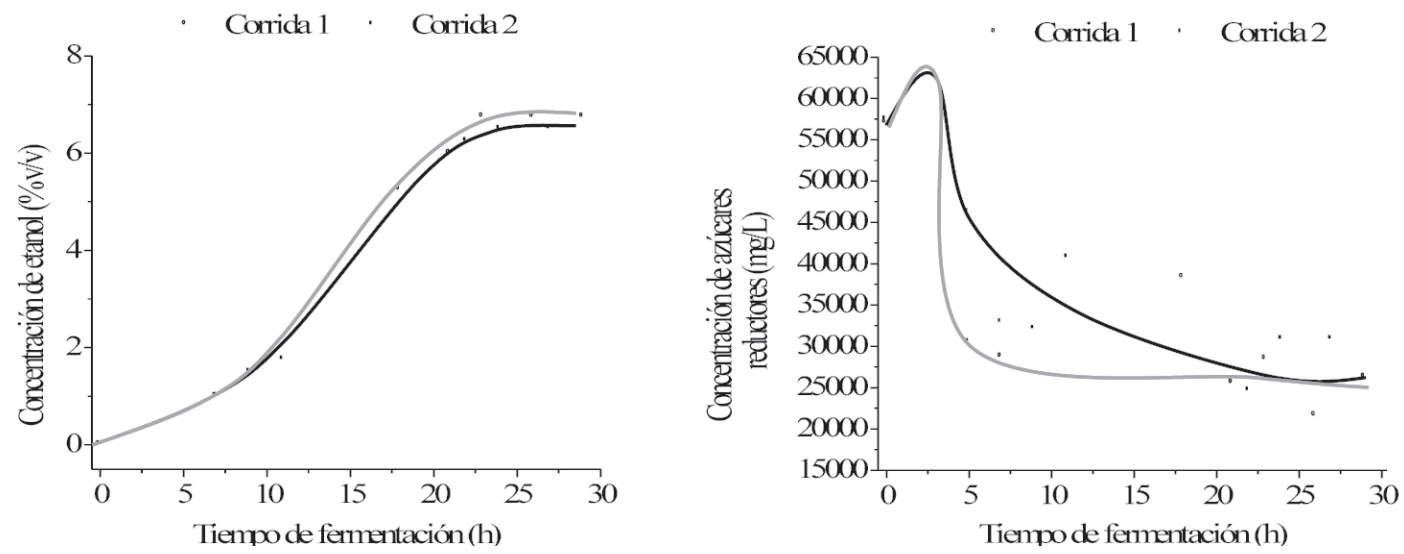

Figura 2. Variación en la concentración de azúcares reductores y concentración de etanol versus tiempo, para la hidrólisis y fermentación realizadas en un reactor de $5 \mathrm{~L}$ 
3. Tanto la temperatura como el tiempo de sacarificación, proporcionan resultados significativamente diferentes en sus distintos niveles, por lo que son factores muy importantes en el proceso de hidrólisis enzimática.

4. Para aumentar el rendimiento de azúcares reductores, la mejor combinación de niveles es una temperatura de sacarificación de 60 ${ }^{\circ} \mathrm{C}$ y un tiempo sacarificación de $2 \mathrm{~h}$.

5. La capacidad de las enzimas $\alpha$-amilasa y AMG para producir azúcares reductores no se ve afectada al adicionar nutrientes durante la hidrólisis enzimática del almidón de sagú.

6. Los resultados de concentración de etanol obtenidos son comparables con valores de concentración de $(6,0$ a 7,5$) \%$ v/v usuales en la industria.

7. La sacarificación puede llevarse a cabo paralelamente a la fermentación, aún cuando se opere a $32^{\circ} \mathrm{C}$.

8. Se recomienda realizar el proceso con las mejores condiciones encontradas $\left(60{ }^{\circ} \mathrm{C}, 2\right.$ h) a nivel de planta piloto, y evaluar otros aspectos tales como agitación y transferencia de calor.

9. Para mejorar los niveles de concentración de etanol se recomienda realizar el crecimiento del microorganismo en un medio igual al sustrato hidrolizado a fermentar para evitar el tiempo de adaptación al medio por parte de la levadura y trabajar con una concentración de levadura alta (50-70 millones $/ \mathrm{mL}$ ).

10. Se recomienda utilizar un sistema totalmente hermético al cual se le haya evacuado el oxígeno mediante el burbujeo de nitrógeno al medio, con el fin de comprobar si se da una mayor producción de etanol por parte del microorganismo.

\section{REFERENCIAS BIBLIOGRÁFICAS}

Anónimo. (2007). AMG 300L y Termamyl 120 L Product Sheet. Novo Nordisk, Dinamarca

Assis, D., Paiva, F., Cabello, C., Dias, R. A. (2010).Energetic study of ethanol production from the corn crops. Ciência Rural, 40 (9), 2017-2022.
Castaño, H., Cardona, M., Mejía, C., Acosta, A. (2011). Producción de etanol a partir de harina de yuca en un sistema de hidrólisis enzimática y fermentación simultánea. Dyna, 78 (169), 158-166.

Cubero, A. M. (2005). Diseño Conceptual de la producción de etanol utilizando sorgo. (Tesis de Licenciatura en Ingeniería Química) Universidad de Costa Rica, San José, Costa Rica.

Cunningham, R., López, G. (1994). Etanol de lignocelulósicos: tecnología y perspectivas. Santa Fe, Argentina, Universidad Santiago de Compostela.

Curvelo-Santana,J.C., Ehrhardt,D.D., Tambourgi, E.B. (2010). Optimizing of alcohol production from manioc starch. Ciência e Tecnologia de Alimentos, 30 (3), 613-617.

Duvernay, W., Chinn, M., Yencho, C. (2013). Hydrolysis and fermentation of sweetpotatoes for production of fermentable sugars and ethanol. Industrial Crops and Products, 42, 527- 537.

González, F. J, Molina, C.M. (2006). Estudio de los factores que afectan la hidrólisis enzimática y el proceso fermentativo para la producción de alcohol a partir de papa. Revista Ingeniería, 16 (1), 29-39.

Harrison, I. (2007). Biocombustibles: Hacia un nuevo modelo de energía alternativa. Ministerio del Ambiente y Energía. Revista Energía. 12. San José, Costa Rica.

Kiliç, D. (2004). $\alpha$-Amylase inactivation during rice starch hydrolysis. Yildiz, Technical University.

López, C.A., Molina, C.M., Huguet, S. A. (2004). Estudio comparativo de la producción de etanol vía fermentativa utilizando cuatro sustratos preparados a partir de banano maduro. Revista Ingeniería, 14 (1,2), 67-77.

Masiero, S. S., Peretti, A., Trierweiler, L., Tierweiler, J. (2014). Simultaneous cold hydrolysis and fermentation of fresh sweet potato. Biomass and Bioenergy, 70, 174-183.

Meléndez, McF. I. (2002). Estudio de la hidrólisis enzimática y del proceso fermentativo para la producción de alcohol a partir de yuca, ñame y malanga. (Tesis de Licenciatura en Ingeniería Química) Universidad de Costa Rica, San José, Costa Rica. 
Monsalve, Medina y Ruiza, J. F., Medina, V., Ruiz, A. (2006). Producción de etanol a partir de la cáscara de banano y de almidón de yuca. Dyna, 73 (150), 21-27.

Sasaki, C., Kushiki, Y., Asada, C., Nakamura, Y. (2014). Acetone-butanol-ethanol production by separate hydrolysis and fermentation (SHF) and simultaneous saccharification and fermentation (SSF) methods using acorns and wood chips of Quercus acutissima as a carbon source. Industrial Crops and Products, 62, 286-292.

\section{SOBRE LOS AUTORES}

Bárbara Miranda Morales. Docente de la Escuela Ingeniería Química, Universidad de Costa Rica. Máster en Ingeniería Química con énfasis en Procesos Industriales. Actualmente es estudiante de Doctorado en la Universidad Rovira i Virgili Correo electrónico: bmiranda34@gmail.com

Manuel Molina Córdoba. Licenciado en Ingeniería Química. Docente de la Escuela de Ingeniería Quimica, Universidad de Costa Rica. 
